\title{
Modeling the Electromyogram (EMG) of Patients Undergoing Anesthesia During Surgery
}

\author{
José Luis Casteleiro-Roca, Juan Albino Méndez Pérez, Andrés José \\ Piñón-Pazos, José Luis Calvo-Rolle and Emilio Corchado
}

\begin{abstract}
All fields of science have advanced and still advance significantly. One of the facts that contributes positively is the synergy between areas. In this case, the present research shows the Electromyogram (EMG) modeling of patients undergoing to anesthesia during surgery. With the aim of predicting the patient EMG signal, a model that allows to know its performance from the Bispectral Index (BIS) and the Propofol infusion rate has been developed. The proposal has been achieved by using clustering combined with regression techniques and using a real dataset obtained from patients undergoing to anesthesia during surgeries. Finally, the created model has been tested with very satisfactory results.
\end{abstract}

Keywords $\mathrm{EMG} \cdot \mathrm{BIS} \cdot$ Clustering $\cdot \mathrm{SOM} \cdot \mathrm{MLP} \cdot \mathrm{SVM}$

\section{Introduction}

One of the facts that has contributed to the outstanding development in some scientific fields is related to the existing synergy with other disciplines. Probably, the most representative examples are the sciences and the techniques around the medicine topics. Typical examples are: robotics, instrumentation, materials and so on.

The present research shows a new advance in this sense, specifically with the Electromyogram signal (EMG) when a patient is undergoing surgery with general

J.L. Casteleiro-Roca · A.J. Piñón-Pazos · J.L. Calvo-Rolle (四)

Departamento de Ingeniería Industrial, University of A Coruña,

Avda. 19 de Febrero s/n, 15495, Ferrol, A Coruña, Spain

e-mail: jose.luis.casteleiro@udc.es

J.A.M. Pérez

Dpto. de Ingeniería de Sistemas y Automática y Arquitectura y

Tecnología de Computadores, University of La Laguna, Avda. Astrof.

Francisco Sánchez s/n, 38200 Santa Cruz de Tenerife, Spain

E. Corchado

Departamento de Informática y Automática, Universidad de Salamanca,

Plaza de la Merced s/n, 37008 Salamanca, Spain

(C) Springer International Publishing Switzerland 2015

Á. Herrero et al. (eds.), 10th International Conference on Soft Computing Models

in Industrial and Environmental Applications, Advances in Intelligent Systems

and Computing 368, DOI 10.1007/978-3-319-19719-7_24 
anesthesia. This research is focused on general anesthesia with Propofol. The measurement of the hypnotic state is done with the Bispectral Index (BIS). This index varies between 0 (no electrical activity) and 100 (awake state). The target for general anesthesia is normally established in 50. The hypothesis in this work is that EMG is correlated to BIS and infusion rate. Then, the objective is to predict the EMG value in terms of BIS and Propofol rate. The EMG signal is used by the clinician to assess the muscular relaxation of the patient [1-3].

For the EMG signal prediction, many different methods can be considered. The common regression methods are typically based on Multiple Regression Analysis (MRA) techniques, that are very common in applications in different fields [4-6]. However, these methods have limitations and do not provide a good performance [5]. In order to increase this feature, many new proposals have been developed. These proposals are based on Soft Computing techniques, both simple or hybrid. As it is shown in [4, 7-20] these techniques improve the first ones mentioned above.

This study implements a hybrid model to predict the EMG signal from the BIS signal and the Propofol infusion rate. To develop the model, K-means clustering algorithm is used to create groups of data with similar behavior. Previously, a SelfOrganization Map (SOM) is employed with the aim of obtaining a first estimation of the optimal clusters quantity. Then, several regression methods were verified for each group to select the best one based on the lowest Mean Squared Error (MSE) reached.

This paper is structured in the following way. After the present section, the case of study is described, the Bispectral index. Then, the model approach and the tested algorithms taken into account in the research are shown. The results section shows the best configuration achieved by the hybrid model. After the results, the conclusions and future works are presented.

\section{Case of Study}

When a patient is undergoing surgery with general anesthesia, a proper dose of Propofol should be administrated to achieve an adequate hypnosis level [21]. To monitor the anesthesia level, the Bispectral Index Signal (BIS) is measured [22, 23]. As a consequence of the anesthesia level and the BIS signal, the ElectroMyoGram signal (EMG) varies according them [23]. The studied problem could be represented as shown in Fig. 1.

The patient is under anesthesia while the right dose of drug is administrated. When the surgery starts the patient is wake up, and then, with the Propofol drug help, is achieved the adequate hypnosis level. During the procedure, the provide level of drug $(\mathrm{mg} / \mathrm{Kg} / \mathrm{h})$ is controlled to achieve the BIS desire level (50). Also, the EMG signal (Volts) depends on the drug quantity. At the end of the surgery the administration of the Propofol is stopped, consequently, the patient wakes up. 


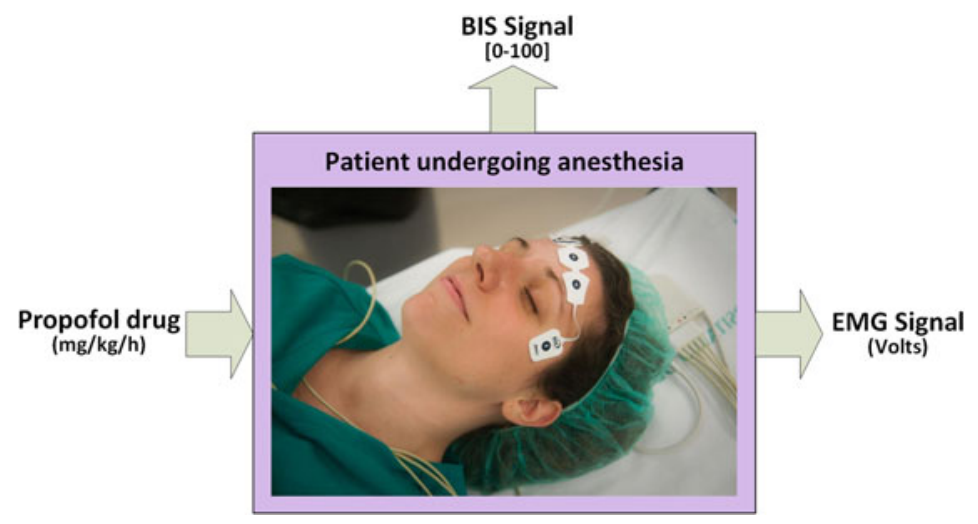

Fig. 1 Case of study. Input/Output representation

\section{Model Approach}

The scheme defined for the model approach is shown in Fig. 2. Taking into account the system behavior and the test accomplished, it is possible to divide the dataset in several operation ranges. Consequently, some clusters are created, and for each one, a regression model is implemented for the single output. As shown in Fig. 2, the global model has two inputs (the -Propofol-drug and the Bispectral index -BIS-) and one output (Electromyogram signal $-E M G-$ ). The cluster selector block connects the chosen models with the output. On each figure cluster block, only the best model is implemented; the cluster for a specific input is selected based on the Euclidean distance between the input and the centroids on each cluster.

The modeling process is shown in Fig. 3. Despite that the figure only shows the data division for training and testing, the dataset has been processed by using crossvalidation (holdout) to ensure the best results for the achieved model.

\subsection{The Dataset Obtaining and Description}

The dataset has been obtained from several patients undergoing general anesthesia with Propofol drug during surgery. The three variables employed on this research (BIS, EMG and Propofol infusion rate) have been monitored during surgeries. A preconditioning stage was considered for BIS and EMG. The dataset is composed with the data of a total of xx patient, recording new set of values every 5 seconds. Due to the signals vary slowly at the acquisition phase, a low pass filter was implemented to avoid the measurement noise. The induction phase and the recovery phase were not considered in this study. Thus, the results obtained are only valid to predict EMG in the maintenance phase of surgery. With the conditions exposed above, the employed dataset contains 2788 samples. 


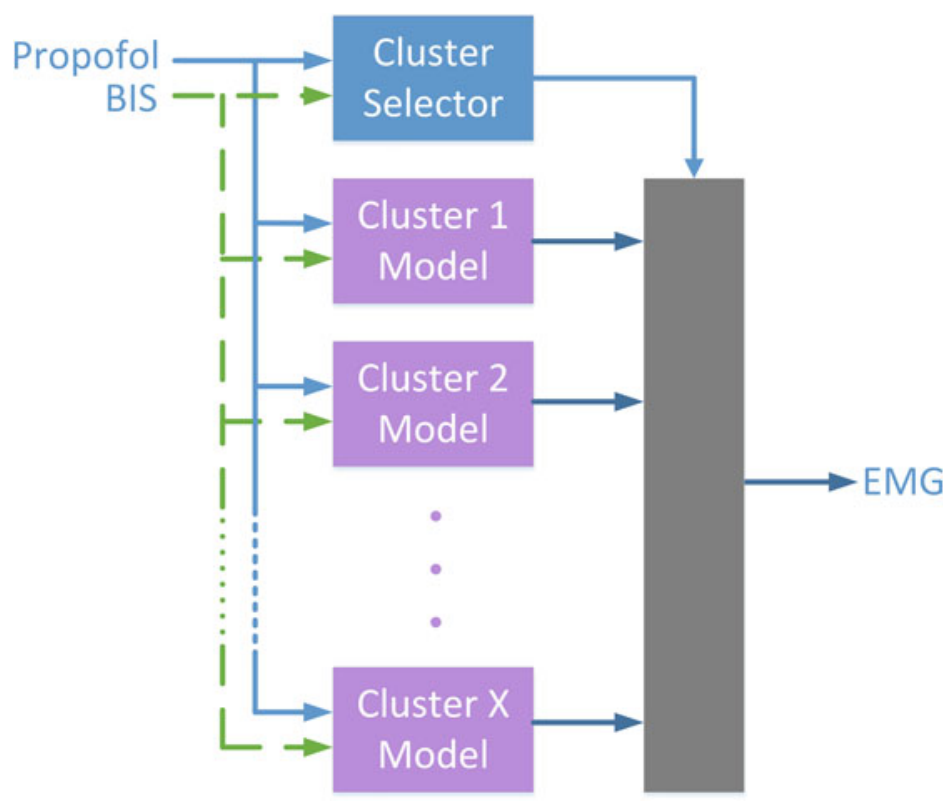

Fig. 2 Model approach

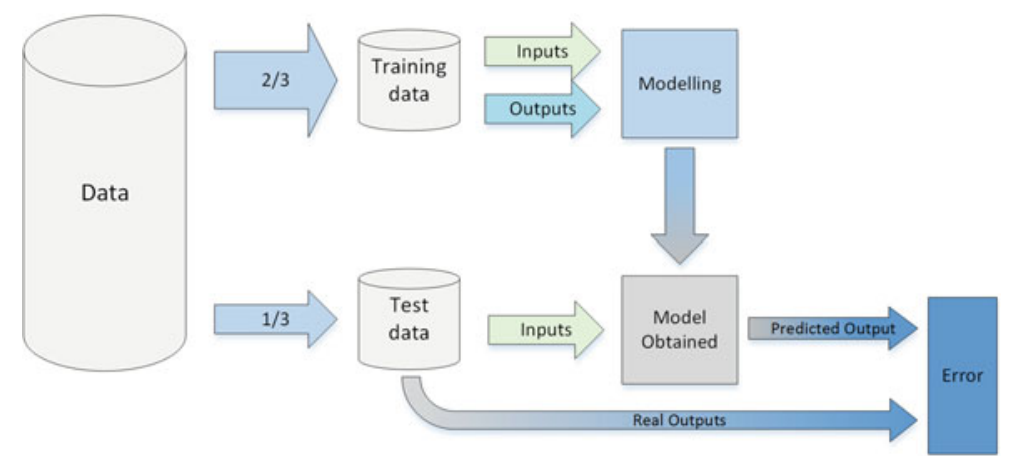

Fig. 3 Modeling process

\subsection{Used Techniques}

The techniques tested in the present study, with the aim of achieving the best model, are described below.

The procedure to obtain the hybrid model is through clustering by using the SOM and K-means algorithms. Below, different intelligent regression techniques are contemplated to accomplish the models. Then, only the best one is chosen according with the MSE criteria. 
Self-Organizing Map (SOM) Self-Organizing Map (SOM) [24] is a type of Artificial Neural Network (ANN) that maps a high dimensionality vector onto a low dimensional one. This type of ANN uses unsupervised competitive learning [25, 26]. The learning process is recurrent until the network reaches a precise result or a maximum number of iterations have been finished. Its configuration is based on an array tied to $\mathrm{N}$ inputs by an $\mathrm{N}$-dimensional weight vector. The process provides the geometry of the data. As a result, a $2 D$ graphical representation is possible to show. Then, possible relationships are discovered by comparing different component maps with another one. The method provides an idea of the number of the required clusters.

Data Clustering - the K-means algorithm Clustering techniques are procedures of data grouping where similarity is measured [27, 28]. These algorithms try to organize unlabeled feature vectors into groups, such samples within a cluster are similar to each other [28]. The K-means method is a frequently used clustering algorithm with square-error criterion, which minimizes error function.

The clustering will depend on the initial cluster centroids and on the $\mathrm{K}$ value (number of groups). The $\mathrm{K}$ value choice is the most critical election because it needs certain previous knowledge of the quantity of clusters present in the data, which is extremely uncertain. The K-means clustering algorithm is computationally effective, it works well if the data are close to its cluster, the cluster is hyperspherical in shape and well-separated in the hyperspace.

Artificial Neural Networks (ANN). Multi-Layer Perceptron (MLP) A MultiLayer Perceptron (MLP) is a feedforward Artificial Neural Network (ANN) [29, 30]. It is one of the most used ANNs due to its simple configuration and its robustness. In spite of this fact, the ANN architecture must be carefully chosen in order to achieve satisfactory results. MLP is made up by one input layer, one or more hidden layers and one output layer. Each layer has neurons, with an activation function. In a typical configuration, all layer neurons have the same activation function. This function could be a step, linear, log-sigmoid or tan-sigmoid.

Support Vector Regression (SVR), Least Square Support Vector Regression (LS-SVR) Support Vector Regression (SVR) is based on the algorithm of the Support Vector Machines (SVM) for classification. In SVR the main aim is mapping the data into a high-dimensional feature space $F$ through a nonlinear plotting and doing linear regression in this space [31].

The Least Square algorithm of SVM is called LS-SVM. The solution estimation is obtained by solving a system of linear equations, and it is similar to SVM in terms of performance generalization [28]. The use of LS-SVM algorithm to regression is well-known as LS-SVR (Least Square Support Vector Regression) [32]. In LS-SVR, the insensitive loss function is replace by a classical squared loss function, which makes the Lagrangian by solving a linear Karush-Kuhn-Tucker (KKT).

Polynomial Regression Usually, a polynomial regression model [33-36] could also be defined as a linear summation of basis functions. The quantity of basis functions depends on the number of inputs of the system, and the degree of the employed polynomial. The model becomes more complex when the degree rises. 


\section{Results}

The model was obtained using the current value of BIS signal, and the Propofol infusion rate quantity of drug (Propofol). To include the dynamic of the modeled system, the last three previous values of the inputs were included to train the models; also, the previous values of the desired output (the EMG signal) were included.

Figure 4 shows one of the results of the SOM analysis of the dataset. In this figure, different regions in the dataset structure should be appreciated. It is necessary to remind that the SOM neighbor weight distances represents the border of different clusters in the dataset with dark lines.

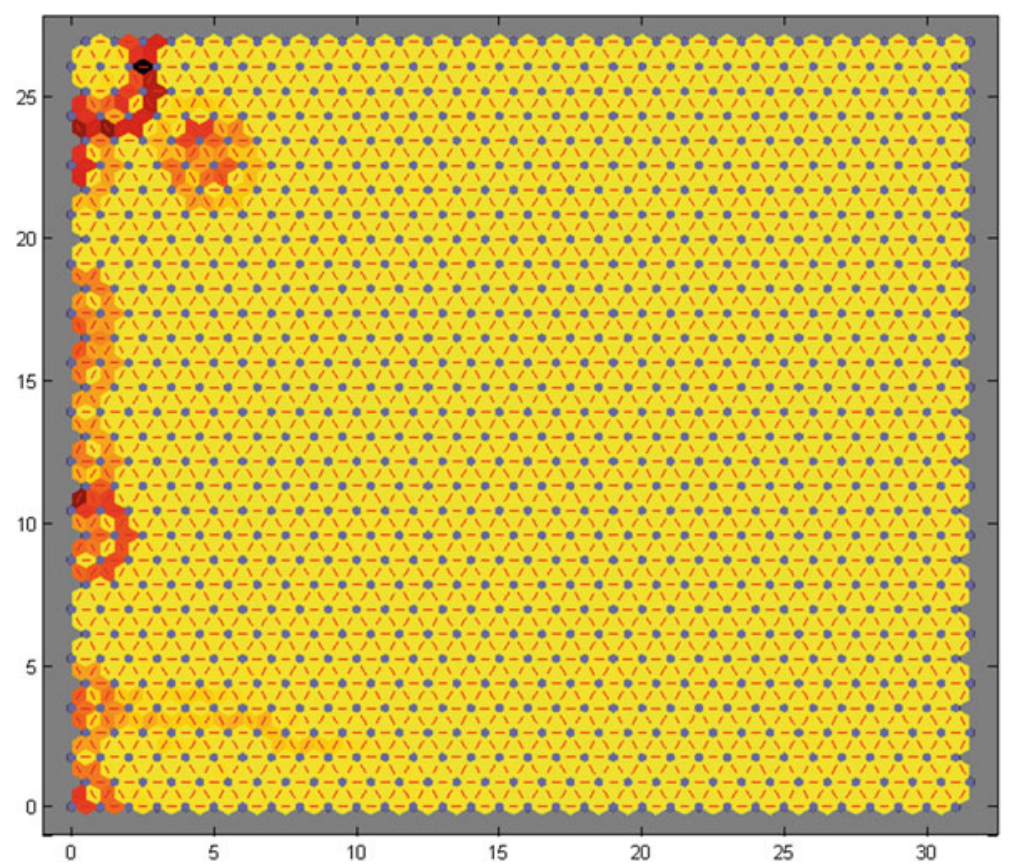

Fig. 4 SOM analysis: Neighbor weight distances

The SOM analysis is very helpful to decided the number of clusters in a dataset when this clusters are well defined. However, in this dataset the clusters are not valid to use the method. As the number of clusters is not previously known, the model was trained with different configuration of clusters, created by using the K-means algorithm.

In each cluster, using cross-validation, $2 / 3$ of the samples were used to train the models, and the other samples were used to calculate the MSE to select the best one. The algorithm performance depends on the initial state, the process was performed 20 times with a random initialization, and finally the best result was stored. The 
K-means configuration was ranged from 2 to 10 clusters with the aim to create 9 different topologies. The global model was taking into account too.

The MLP-ANN regression algorithm was trained for different configurations; always with one hidden layer, but the number of neurons in the hidden layer varies from 2 to 15. The activation function of this neurons was tan-sigmoid for all tests, and the output layer neuron had a linear activation function. The training algorithm used was Levenberg-Marquardt; gradient descent was used as learning algorithm, and the performance function was set to mean squared error.

The LS-SVR was trained with the self-tuning implemented with the toolbox for MatLab developed by KULeuven-ESAT-SCD. The kernel of the model was set to Radial Basis Function (RBF), and the type was 'Function Estimation' to perform regression. The optimization function is 'simplex' and the cost-criterion is 'leaveoneoutlssvm' with 'mse' as a performance function.

For Polynomial regression, the order of the polynomial trained varies from $1^{\text {st }}$ to $20^{\text {th }}$ order.

Table 1 shows the best MSE achieved for each cluster with the corresponding test data(with all the different configurations tested). Moreover, in the last column is shown the worst MSE achieved for each configuration. The best hybrid model was chosen taking into account the worst cases, and then selecting the best.

Table 2 shows the best regression technique used and its configuration for each cluster.

The best configuration achieved for the model was the one that divides the data in 2 different clusters, as is shown in Table 3. In this table, the best configuration for each algorithm is presented. Each cluster named was based on the quantity of the samples (2763 samples in the big cluster, and only 25 in the small cluster).

\section{Conclusions}

This study provides a precise way of modeling the Electromyogram (EMG). The accomplished model predicts the EMG from the Bispectral index (BIS) signal and the Propofol drug quantity provided to the patient

This model was obtained from a real dataset. The approach is based on a hybrid intelligent system, by combining different regression techniques on local models. After some tests, the analysis of the results shows that the best model configuration has 2 clusters. The regression techniques employed on the clusters were ANN with different configurations ( 5 and 3 neurons in the hidden layer). The best average MSE obtained with this configuration was 1.1083 . 
Table 1 Best MSE for each cluster

\begin{tabular}{|c|c|c|c|c|c|c|}
\hline $\begin{array}{l}\mathrm{N}^{\circ} \text { of } \\
\text { Clusters }\end{array}$ & \begin{tabular}{|l} 
Cluster \\
1
\end{tabular} & \begin{tabular}{|l} 
Cluster \\
2
\end{tabular} & $\begin{array}{l}\text { Cluster } \\
3\end{array}$ & $\begin{array}{l}\text { Cluster } \\
4\end{array}$ & $\begin{array}{l}\text { Cluster } \\
5\end{array}$ & $\begin{array}{l}\text { Clusters } \\
\text { MSE }\end{array}$ \\
\hline $\begin{array}{l}\text { Global } \\
\text { Model }\end{array}$ & 1.4702 & - & - & - & - & 1.4702 \\
\hline 2 Clusters & 1.1083 & 0.0118 & - & - & - & 1.1083 \\
\hline 3 Clusters & 3.0819 & 1.1470 & 1.2154 & - & - & 3.0819 \\
\hline 4 Clusters & 0.8627 & 0.9678 & 12.1164 & 17.3731 & - & 17.3731 \\
\hline 5 Clusters & 0.6532 & 0.2730 & 38.1696 & 0.5758 & 0.8678 & 38.1696 \\
\hline 6 Clusters & 0.6307 & 1.3774 & 0.6544 & 1.8068 & 17.6927 & 17.6927 \\
\hline 7 Clusters & 2.8374 & 0.5853 & 0.8183 & 0.8000 & 17.0372 & 17.0372 \\
\hline 8 Clusters & 1.0466 & 1.2601 & 1.8061 & 0.5447 & 1.0011 & 24.9626 \\
\hline 9 Clusters & 2.3795 & 53.3880 & 1.2450 & 22.6021 & 0.0092 & 53.3880 \\
\hline 10 Clusters & 0.4696 & 18.1680 & 0.3254 & 0.9348 & 0.0173 & 18.1680 \\
\hline $\begin{array}{l}\mathrm{N}^{\circ} \text { of } \\
\text { Clusters }\end{array}$ & $\begin{array}{l}\text { Cluster } \\
6\end{array}$ & $\begin{array}{l}\text { Cluster } \\
7\end{array}$ & $\begin{array}{l}\text { Cluster } \\
8\end{array}$ & $\begin{array}{l}\text { Cluster } \\
9\end{array}$ & $\begin{array}{l}\text { Cluster } \\
10\end{array}$ & $\begin{array}{l}\text { Clusters } \\
\text { MSE }\end{array}$ \\
\hline $\begin{array}{l}\text { Global } \\
\text { Model }\end{array}$ & - & - & - & - & - & 1.4702 \\
\hline 2 Clusters & - & - & - & - & - & 1.1083 \\
\hline 3 Clusters & - & - & - & - & - & 3.0819 \\
\hline 4 Clusters & - & - & - & - & - & 17.3731 \\
\hline 5 Clusters & - & - & - & - & - & 38.1696 \\
\hline 6 Clusters & 0.4948 & - & - & - & - & 17.6927 \\
\hline 7 Clusters & 0.0055 & 0.8909 & - & - & - & 17.0372 \\
\hline 8 Clusters & 24.9626 & 0.8936 & 0.0020 & - & - & 24.9626 \\
\hline 9 Clusters & 1.1125 & 0.4152 & 1.2255 & 1.9976 & - & 53.3880 \\
\hline 10 Clusters & 0.8402 & 1.1774 & 1.5591 & 0.0177 & 0.0966 & 18.1680 \\
\hline
\end{tabular}

This analysis could be applied to several different systems with the aim of improving other specifications like: efficiency, performance, features of the obtained material. It is important to emphasize that quite satisfactory results have been obtained with the approach proposed in this research. 
Table 2 Best regression technique for each cluster

\begin{tabular}{l|l|l|l|l|l}
\hline $\begin{array}{l}N^{\circ} \text { of } \\
\text { Clusters }\end{array}$ & 1 & Cluster & 2 & $\begin{array}{l}\text { Cluster } \\
\text { Cluster }\end{array}$ & $\begin{array}{l}\text { Cluster } \\
5\end{array}$ \\
\hline \hline Global Model & Poly-04 & - & - & - & - \\
\hline 2 Clusters & ANN-05 & ANN-03 & - & - & - \\
\hline 3 Clusters & ANN-03 & Poly-02 & LS-SVR & - & - \\
\hline 4 Clusters & LS-SVR & LS-SVR & ANN-04 & LS-SVR & - \\
\hline 5 Clusters & LS-SVR & ANN-06 & Poly-01 & LS-SVR & ANN-04 \\
\hline 6 Clusters & ANN-08 & Poly-01 & LS-SVR & ANN-02 & Poly-01 \\
\hline 7 Clusters & ANN-02 & ANN-07 & LS-SVR & ANN-05 & Poly-09 \\
\hline 8 Clusters & Poly-03 & ANN-07 & LS-SVR & Poly-06 & ANN-06 \\
\hline 9 Clusters & Poly-01 & ANN-15 & ANN-11 & Poly-01 & ANN-05 \\
\hline 10 Clusters & LS-SVR & Poly-01 & Poly-01 & LS-SVR & ANN-05 \\
\hline N of & Cluster & Cluster & Cluster & Cluster & Cluster \\
Clusters & 6 & 7 & 8 & 9 & 10 \\
\hline \hline Global Model & - & - & - & - & - \\
\hline 2 Clusters & - & - & - & - & - \\
\hline 3 Clusters & - & - & - & - & - \\
\hline 4 Clusters & - & - & - & - & - \\
\hline 5 Clusters & - & - & - & - & - \\
\hline 6 Clusters & LS-SVR & - & - & - & - \\
\hline 7 Clusters & ANN-15 & LS-SVR & - & - & - \\
\hline 8 Clusters & ANN-02 & LS-SVR & ANN-03 & - & - \\
\hline 9 Clusters & LS-SVR & Poly-02 & ANN-13 & Poly-01 & - \\
\hline 10 Clusters & ANN-03 & LS-SVR & Poly-08 & ANN-13 & ANN-14 \\
\hline
\end{tabular}

Table 3 Best result for the final configuration of the proposal

\begin{tabular}{l|l|l}
\hline & Big Cluster & Small Cluster \\
\hline Train samples & 1842 & 17 \\
\hline Test samples & 921 & 8 \\
\hline Best ANN & 5 neurons & 3 neurons \\
\hline Best Polynomial & First order & First order \\
\hline \hline ANN MSE & 1.1083 & 0.0118 \\
\hline LSSVM MSE & 1.6241 & 6.9461 \\
\hline Poly MSE & 1.3546 & 58.5147 \\
\hline
\end{tabular}


Acknowledgments This study was conducted under the auspices of Research Project DPI2010 18278, supported by the Spanish Ministry of Innovation and Science.

\section{References}

1. Coelho AL, Lima CA (2014) Assessing fractal dimension methods as feature extractors for EMG signal classification. Eng Appl Artif Intell 36:81-98

2. Xing K, Yang P, Huang J, Wang Y, Zhu Q (2014) A real-time EMG pattern recognition method for virtual myoelectric hand control. Neurocomputing 136:345-355

3. Phinyomark A, Quaine F, Charbonnier S, Serviere C, Tarpin-Bernard F, Laurillau Y (2013) A feasibility study on the use of anthropometric variables to make musclecomputer interface more practical. Eng App Artif Intell 26(7):1681-1688

4. Ghanghermeh A, Roshan G, Orosa JA, Calvo-Rolle JL, Costa ÁM (2013) New climatic indicators for improving urban sprawl: a case study of tehran city. Entropy 15(3):999-1013

5. Calvo-Rolle JL, Quintian-Pardo H, Corchado E, Del Carmen Meizoso-López M, García RF (2015) Simplified method based on an intelligent model to obtain the extinction angle of the current for a single-phase half wave controlled rectifier with resistive and inductive load. J Appl Logic 13(1):37-47

6. Calvo-Rolle JL, Fontenla-Romero O, Pérez-Sánchez B, Guijarro-Berdinas B (2014) Adaptive inverse control using an online learning algorithm for neural networks. Informatica 25(3):401414

7. García RF, Rolle JLC, Gomez MR, Catoira AD (2013) Expert condition monitoring on hydrostatic self-levitating bearings. Expert Syst Appl 40(8):2975-2984

8. Calvo-Rolle JL, Casteleiro-Roca JL, Quintián H, Del Carmen Meizoso-Lopez M (2013) A hybrid intelligent system for PID controller using in a steel rolling process. Expert Syst Appl 40(13):5188-5196

9. García RF, Rolle JLC, Castelo JP, Gomez MR (2014) On the monitoring task of solar thermal fluid transfer systems using NN based models and rule based techniques. Eng Appl Artif Intell 27:129-136

10. Quintián H, Calvo-Rolle JL, Corchado E (2014) A hybrid regression system based on local models for solar energy prediction. Informatica 25(2):265-282

11. Nieto PG, Torres JM, de Cos Juez F, Lasheras FS (2012) Using multivariate adaptive regression splines and multilayer perceptron networks to evaluate paper manufactured using eucalyptus globulus. Appl Math Comput 219(2):755-763

12. de Cos Juez F, Nieto PG, Torres JM, Castro JT (2010) Analysis of lead times of metallic components in the aerospace industry through a supported vector machine model. Math Comput Model 52(78):1177-1184 (Mathematical Models in Medicine, Business and Engineering 2009)

13. de Cos J, Sanchez F, Ortega F, Montequin V (2008) Rapid cost estimation of metallic components for the aerospace industry. Int J Prod Econ 112(1):470-482 (Special Section on Recent Developments in the Design, Control, Planning and Scheduling of Productive Systems)

14. Casteleiro-Roca J, Calvo-Rolle J, Meizoso-López M, Piñón-Pazos A, Rodríguez-Gómez B (2015) Bio-inspired model of ground temperature behavior on the horizontal geothermal exchanger of an installation based on a heat pump. Neurocomputing 150 Part A(0):90-98

15. Calvo-Rolle JL, Machón-González I, López-García H (2011) Neuro-robust controller for nonlinear systems. DYNA 86(3):308-317

16. Quintián-Pardo H, Calvo-Rolle JL, Fontenla-Romero O (2012) Application of a low cost commercial robot in tasks of tracking of objects. Dyna 175:24-33

17. Vilar-Martínez XM, Montero-Sousa JA, Calvo-Rolle JL, Casteleiro-Roca JL (2014) Expert system development to assist on the verification of TACAN system performance. DYNA 89(1):112-121 
18. Garcia RF, Calvo-Rolle JL, Gomez MR, Catoira AD (2013) Expert condition monitoring on hydrostatic self-levitating bearings. Expert Syst Appl 40(8):2975-2984

19. Garcia RF, Calvo-Rolle JL, Castelo JP, Gomez MR (2014) On the monitoring task of solar thermal fluid transfer systems using NN based models and rule based techniques. Eng Appl Artif Intell 27:129-136

20. Machon-Gonzalez I, Lopez-Garcia H, Calvo-Rolle J (2010) A hybrid batch som-ng algorithm. In: The 2010 International Joint Conference on Neural Networks (IJCNN), pp 1-5

21. Litvan H, Jensen EW, Galan J, Lund J, Rodriguez BE, Henneberg SW, Caminal P, Villar Landeira JM (2002) Comparison of conventional averaged and rapid averaged, autoregressivebased extracted auditory evoked potentials for monitoring the hypnotic level during propofol induction. J Am Soc Anesthesiologists 97(2):351-358

22. Sánchez SS, Vivas AM, Obregón JS, Ortega MR, Jambrina CC, Marco ILT, Jorge EC (2009) Monitorización de la sedación profunda. el monitor BIS. Enfermería Intensiva 20(4):159-166

23. Pérez JAM, Torres S, Reboso JA, Reboso H (2011) Estrategias de control en la práctica de anestesia. Rev Iberoamericana de Automática e Informática Ind RIAI 8(3):241-249

24. Kohonen T (1990) The self-organizing map. Proc IEEE 78(9):1464-1480

25. Kohonen T, Oja E, Simula O, Visa A, Kangas J (1996) Engineering applications of the selforganizing map. Proc IEEE 84(10):1358-1384

26. Kohonen T (1997) Exploration of very large databases by self-organizing maps. In: International Conference on Neural Networks, vol 1, pp PL1-PL6

27. Qin A, Suganthan P (2005) Enhanced neural gas network for prototype-based clustering. Pattern Recogn 38(8):1275-1288

28. Kaski S, Sinkkonen J, Klami A (2005) Discriminative clustering. Neurocomputing 69(13):1841

29. Wasserman P (1993) Advanced Methods in Neural Computing, 1st edn. Wiley, New York

30. Zeng Z, Wang J (2010) Advances in Neural Network Research and Applications, 1st edn. Springer Publishing Company, New York

31. Vapnik V (1995) The Nature of Statistical Learning Theory. Springer, Berlin (1995)

32. Li Y, Shao X, Cai W (2007) A consensus least squares support vector regression (LS-SVR) for analysis of near-infrared spectra of plant samples. Talanta 72(1):217-222

33. Heiberger R, Neuwirth E (2009) Polynomial regression. In: R Through Excel. Use R, pp 269284. Springer New York

34. Casteleiro-Roca J, Calvo-Rolle J, Meizoso-López M, Piñón-Pazos A, Rodríguez-Gómez B (2014) New approach for the QCM sensors characterization. Sens Actuators A: Physical 207: $1-9$

35. Wu X (2007) Optimal designs for segmented polynomial regression models and web-based implementation of optimal design software, pp AAI3337619. State University of New York at Stony Brook, Stony Brook, New York

36. Zhang Z, Chan SC (2011) On kernel selection of multivariate local polynomial modelling and its application to image smoothing and reconstruction. J Signal Process Syst 64(3):361-374 